\title{
The model of UAV formation based on the uniform allocation of points on the sphere
}

\author{
Mikhail Khachumov, Vyacheslav Khachumov \\ Federal Research Center "Computer Science and Control" of the Russian Academy of Sciences, 117312, Moscow, Russia
}

\begin{abstract}
We consider an approach to constructing the desired virtual structure, which should be formed by unmanned aerial vehicles (UAVs). The proposed model is based on the principle of quasi-uniform allocation of points, previously used by the author in clustering problems. The mathematical apparatus for solving the problem of forming the desired structure is given: necessary theorems are proved; the hypothesis on the uniform distribution of points on the boundary of the circle and the sphere is put forward and partially proved. The method for optimal assignment of UAVs to goal positions in the desired formation is considered.
\end{abstract}

\section{Introduction}

Due to the active use of unmanned aerial vehicles (UAVs) in various spheres of human activity, the problem of forming UAV coalitions and using them in solving practical tasks becomes urgent. The general problem of forming a desired formation contains the following steps:

1) Forming the desired topology of homogeneous UAVs in a certain area, i.e. the structure of goal positions. We note the existence of a huge number of possible structures, but we focus on the bounded regular geometric shapes. As a rule, these are regular polygons on the plane and Platonic solids realizing the principle of equidistance for adjacent vertices. Another approach assumes the uniform allocation of points in a given space. The problem is not yet solved and requires the development of algorithms for calculating coordinates for an arbitrary number of points.

2) Planning the motion of UAVs to the goal positions from a given arbitrary location. This problem is called formation or reconfiguration of a given shape. The problem of planning optimal (in terms of time, distance, energy costs, etc.) and at the same time safe UAVs routes with respect to size and velocities is posed. It is needed to define a sequence of controls that lead a group of homogeneous UAVs from the initial position to the desired one [1]. The task is complicated by the presence of wind disturbances that affect the velocities, trajectories, distances between UAVs and, ultimately, the safety of group motion.

The problems of formation and reconfiguration of a given structure for a group of mobile vehicles (robots, drones, aircrafts, etc.) are considered in many state-of-art research papers.

In paper [2] the authors provide an extensive overview of the methods of UAVs allocation, based on the concept of uniform distribution of points on different surfaces. Control and navigation algorithms are proposed for a swarm of autonomous homogeneous mobile robots involved in the formation of a given spatial convex surface. Calculation of robots trajectories for swarm reconfiguring is carried out with minimizing time and energy costs (computation time and total length of trajectories) and with controlling collisions. The developed algorithm has quadratic computational complexity.

In paper [3] the authors proposed a new method for topology design of a group of robots based on a multilevel structure, which is employed to present different shapes of swarm robots in the desired region. A potential function-based controller makes it possible to control the robots in forming the desired formation shape and avoid collisions. The local minima problem that causes the robots to get stuck at undesired positions is solved by using new shape regulation control force. Experiments are performed to demonstrate the effectiveness of the proposed approach.

Formation control strategies for UAVs with fully available group trajectory information are considered in [4]. A unified, distributed architecture for controlling vehicles' layers requires only local neighbour-toneighbour information exchange. A group of robots should form a certain spatial shape. It is proposed to decompose the virtual shape into parts and divide the swarm into several subsets (groups) responsible for the formation of its own part of the shape.

In paper [5] the authors propose formation control of nonholonomic mobile robots avoiding obstacles in a distributed manner for cluttered environments. The asymptotic convergence of follower robots to the position and orientation of the reference robot is ensured using the Lyapunov function. Simulations results are

* Corresponding author: khmike@inbox.ru 
provided demonstrating formation control and obstacle avoidance for multirobots using the proposed scheme.

The problem of forming large groups of individuals moving together toward a common target location is considered in [6]. The possibility of multi-agent technologies application to this problem is studied. Consensus-based algorithm to control a swarm in dynamically changing and noisy environments is proposed.

Basically, all considered papers solve the problem of reaching already established goal points. Coordinates of those points are assumed to be known. In this paper, we solve two particular, but theoretically interesting problems of structure formation when UAVs must form a regular flat or spatial shape that meets the requirements of uniform allocation of goal points.

Earlier we obtain theoretical results in cluster analysis that may be utilised for solving UAV formation problems. The methods and models of uniform distribution of points presented in [7] are applicable to solving the problem of safe formation of desired shapes for UAVs. This article provides an interpretation of these results.

\section{The model of UAVs formation}

We consider the geometrical model of a UAV formation based on the distribution of $n$ points in a sphere. We introduce the following definitions.

Definition 1. The condition of the uniform distribution $m_{\mathrm{i}}(i=1,2, \ldots, n)$ of points on a $p$-sphere in $R^{p}$ is fulfilled if the points are the vertices of regular polyhedrons that are circumscribed by the sphere. The regular $N$ dimensional polyhedron is a convex polyhedron (i.e., the distance between any nearest points is constant and equal to the edge of polyhedron).

Definition 2. The condition of the uniform distribution $m_{\mathrm{i}}(i=1,2, \ldots, n)$ of points on a sphere of radius $r$ in $R^{p}(n>p)$ is fulfilled if for each point $m_{\mathrm{i}}$ exist $p$ other points $m_{\mathrm{jp}}$ such that $d\left(m_{i}, m_{j p}\right)=\operatorname{const}(n, p, r)$.

Definition 3 (Thomson's uniformity). Let us allocate $n$ identical free charges on a sphere. We consider that the distribution is quasi-uniform if it corresponds to the minimum of the potential energy of the system [8].

It follows from Definition 2 that the number of solutions of the problem is bounded by the number of regular polyhedra. Definition 3 does not cover the cases when $n \leq p$, as well as leads to difficulties in constructing an algorithm for calculating the coordinates of uniformly spaced points.

\subsection{The hypothesis of quasi-uniformity of the distribution of points}

Let $m_{\mathrm{i}} \in R^{p}(i=1,2, \cdots, n)$ be the points on the $p$-sphere of radius $r$; let $S$ be the boundary of the $p$-sphere and $d\left(m_{i}, m_{j}\right)$ be the distance between the points $m_{\mathrm{i}}$ and $m_{j}$. Let us propose the following hypothesis about the quasiuniformity of their distribution in a space bounded by a sphere.
Hypothesis. The expression $\sum_{1 \leq i<j \leq n} d\left(m_{i}, m_{j}\right)$ takes the maximum when the points $m_{i}$ are quasi-uniformly located on the border $S$ of a $p$-sphere.

Unfortunately, the proof of Hypothesis for a $p$ dimensional sphere causes some difficulties; thus, it is proposed to consider some particular cases, whose solution has an independent and important value in practice.

\subsubsection{The problem of optimal allocation of points on a plane bounded by a circle}

Let us show that the given Hypothesis is valid for the planar case. Consider model problem 1 (packing problem): how to allocate the points in a circle of radius $r$ such that the sum of the distances is maximal.

Lemma 1 (about the quasi-uniform allocation of points within the circle). Let $m_{i}(i=1,2, \ldots, n)$ be the points that lie on the circle $K(r)$ of radius $r$; let $L$ be the boundary of the circle $K(r)$ and $d\left(m_{i}, m_{j}\right)=d_{i, j}$ be the distance between the points $m_{\mathrm{i}}$ and $m_{j}$. The expression $\sum_{1 \leq i<j \leq n} d\left(m_{i}, m_{j}\right)$ takes the maximum values when the points $m_{i}$ are quasi-uniformly located on the boundary of the circle L. The proof of this lemma is given in [9].

\subsubsection{The problem of optimal allocation of points in a sphere}

The original statements of problems on the sphere are given in [10]. Consider model problem 2: how can one allocate $n$ points on the $p$-sphere of radius $r$ such that the sum of the distances is maximal.

The solution of the problem is the subject of actual research and underlies the predictable geometric allocaton of points [7, 9].

Theorem. Let $m_{\mathrm{i}} \in R^{p}(i=1,2, \cdots, n)$ be the points on the $p$-sphere $K(r)$ of radius $r$, let $S$ be the boundary of the sphere $K(r)$ and $d\left(m_{i}, m_{j}\right)$ be the distance between points $m_{\mathrm{i}}$ and $m_{j}$. Then exist points $m_{i}^{\prime} \in S(i=1,2, \cdots, n)$ such that $\sum_{1 \leq i<j \leq n} d\left(m_{i}, m_{j}\right) \leq \sum_{1 \leq i<j \leq n} d\left(m_{i}^{\prime}, m_{j}^{\prime}\right)$.

Proof.

Let some allocation of the points $m_{i}(i=1,2, \ldots, n)$ exist in $p$ sphere $K(r)$. Show that any point $m_{\mathrm{i}} \in K(r)$ can be shifted to the sphere $S$ so that the value $\sum_{1 \leq i<j \leq n} d\left(m_{i}, m_{j}\right)$

does not decrease. We select the arbitrary point $m_{\mathrm{i}}$ and plot a chord through it. Introduce a new coordinate system, wherein one of the coordinate axes will coincide with the chord. Since $m_{i}$ moves along the chord, its coordinates are $(t ; 0 ; \ldots ; 0)$, while the coordinates of the point are $m_{j}=\left(a_{1} ; a_{2} ; \cdots ; a_{p}\right)$. Then, the function of the parameter $t$, which is equal to the distance from the point $m_{j} \in K(r)$ to the point $m_{i}$ that lies on the chord is $d\left(m_{i}, m_{j}\right)=f(t)=\sqrt{\left(a_{1}-t\right)^{2}+a_{2}{ }^{2}+\ldots+a_{p}{ }^{2}}$.The function $f(t)$ depend only on the variable $t$, as the point $m_{j} \in K(r)$ 
is fixed. The function $f(t)$ is convex since its second derivative is positive.

Let us consider the analogous function for other points. The sum of convex functions $\sum_{j=1}^{n} d\left(m_{i}, m_{j}\right)$ is convex. The maximum value of a convex function is in one of the ends of a segment, i.e., a shift of the point to the sphere does not reduce the values of $\sum_{1 \leq i<j \leq n} d\left(m_{i}, m_{j}\right)$.

Theorem is proved.

Let us consider the case of a three-dimensional sphere.

Lemma 2. Let $m_{i}(i=1,2, \ldots, 4)$ be the points on a three-dimensional sphere. The expression $\sum_{1 \leq i<j \leq 4} d\left(m_{i}, m_{j}\right)$ takes the maximum when the points $m_{i}$ matches the vertices of the regular tetrahedron.

The proof of this lemma is given in [9].

Lemmas 1 and 2 are important and give partial results that confirm the Hypothesis. We note that in both concidered special cases the conditions of Definition 2 are also satisfied, e.i., the distribution of points is uniform.

\subsection{The Thomson model of quasi-uniform placement of points.}

Definition 3 relates to the theoretical justification of the optimality of the configuration of charges considered by the physicist Thomson [8]. The problem is to determine the global minimum potential energy configuration of equal charges constrained to the surface of a sphere (without external influences): $\min U=\sum\left(1 /\left(r_{i}-r_{j}\right)\right), i<j$, where $r_{1}, r_{2}, \ldots, r_{n}$ are radius-vectors of charges (unit charges and vectors are considered).

An iterative algorithm for practical allocation of points on a sphere is developed. Visualization of the results for seven charges is shown in Figure 1.

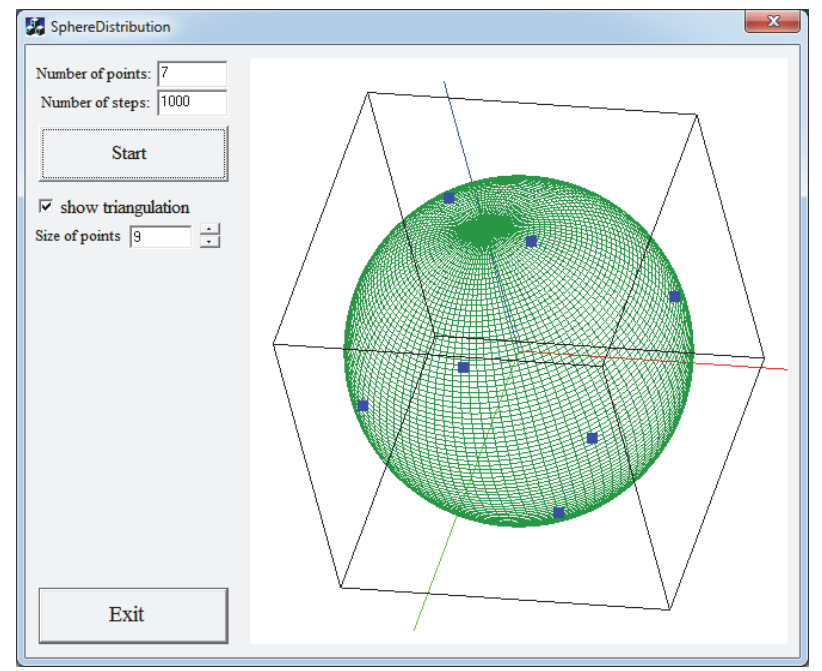

Fig. 1. Quasi-uniform distribution of seven points on the sphere.
If necessary, the algorithm additionally constructs the triangulation mesh. By solving the triangulation problem a reasonable distribution of points can be achieved.

Definition 4. Triangulation is called optimal if the sum of the lengths of all edges is minimal among all possible triangulations constructed on the same initial points. The mesh constructed by the methods of exhaustion, as a rule, have good quality. By optimizing the position of nodes one can achieve better quality.

The Thomson problem in three-dimensional space is solved exactly only for the cases of 2, 3, 4, 6 and 12 charges on a sphere. When the number of charges is 2,3 or 4 , in extreme configuration all pairwise distances between them are equal. For other cases, the optimality of any disposition is not proved.

Note that, for these particular cases the results of placement of points coincide according to Definitions 2 and 3. That confirms the validity of the chosen criterion.

\section{The problem of assignment of UAVs to goal positions}

\subsection{Formulation of the problem}

Assume that we know the goal positions in the desired structure and positions of all UAVs. The problem is to optimally assign UAVs to available points in the formation. The problem can be presented in the form of a bipartite graph (Fig. 2), where vertices of one layer correspond to UAVs $\left\{p_{1}, p_{2}, \ldots, p_{\mathrm{n}}\right\}$ and vertices of another layer correspond to the positions of the formation $\left\{q_{1}, q_{2}, \ldots, q_{n}\right\}$.

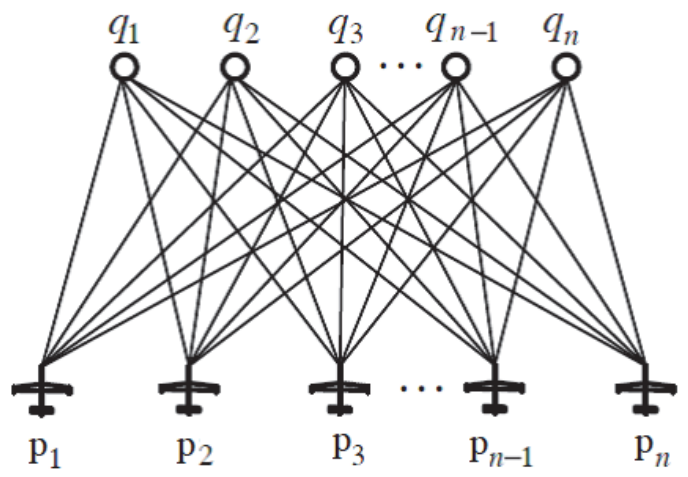

Fig. 2. Bipartite graph of relations "UAV-formation".

The edges of the graph correspond to the elements of the distance matrix $D=\left\|d_{i j}\right\|$, containing information about the distances between UAVs and goal positions. In addition, we assign $d_{i j}=\infty$, if the flight of UAV $i$ to position $j$ is impossible or undesirable.

Costs that correspond to the flight of UAV $i$ to point $j$, we denote as $c_{i j}$. Here, we assume $c_{i j}=d_{i j}$. Alternatively one can use time costs that correspond to the flight of UAV $i$ to point $j$, i.e. $c_{i j}=d_{i j} /\left(v_{i}+v_{i j}\right)(i, j=$ $1,2, \ldots, n)$, where $v_{i}$ is an average speed of UAV $i$; $v_{i j}$ is the speed of the air flow between position of UAV $i$ and goal position $j$. We introduce $x_{i j}(i, j=1,2, \ldots, n)$, such that $x_{i j}=1$ if UAV $i$ is assigned to point $j$, and $x_{i j}=0$ otherwise. 
A typical assignment problem is formulated as a linear programming problem [11]:

$$
c=\sum_{i=1}^{n} \sum_{j=1}^{n} c_{i j} x_{i j} \rightarrow \min ,
$$

where: $\sum_{i=1}^{n} x_{i j}=\sum_{j=1}^{n} x_{i j}=1 \quad \forall i, j=1,2, \ldots, n$.

To solve the problem the Hungarian method can be used [11]. As a result of solving the problem a table is obtained with marked zeros that correspond to the assigned items. A similar approach is concidered in $[12,13]$.

\subsection{Flight safety assurance}

When all UAVs are assigned to goal positions it is necessary to plan their flight trajectories guaranteeing safety. Collision avoidance can be ensured by constructing for each vehicle of a tube (3D curve) [14] determined by circular object. All trajectories within such a tube are safe. The main criteria for optimal selection of tubes are: time of construction and length of the tube. To plan the optimal route one can use special software T-FLEX CAD 3D, which implements the basic operations of $3 \mathrm{D}$ modeling. Alternatively to detect collisions one can use the matrix of the current distances between UAVs. In that case decisions are made by certain rules adopted in aviation. It is planned to carry out further research on the construction of safe trajectories for collisions avoidance.

\section{Experimental researches}

We simulate the problem of forming a structure in a group of UAVs, taking into account real mathematical models of objects and wind disturbances [15]. Figure 3 shows formation flight of four UAVs and demonstrates the effect of wind loading on the motion trajectories.

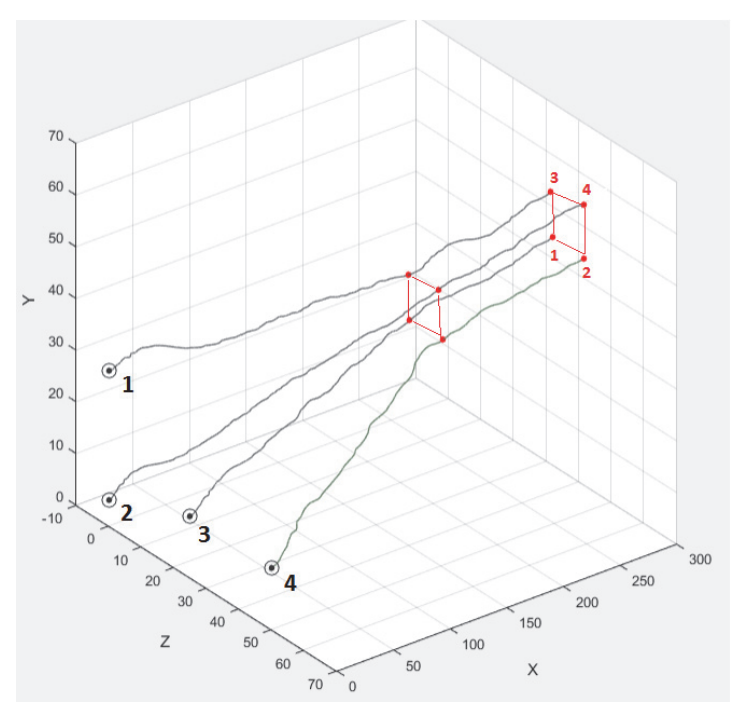

Fig. 3. Formation flight of four UAVs.

It can be seen that vehicles (grey markers) are assigned to uniformly distributed positions (red markers) in the desired formation. The developed simulating system contains a special module of intelligent control, realizing strategies and control rules for prompt response to changes in the external environment.

\section{Conclusion}

The proposed definitions and hypothesis of quasiuniformity of the distribution of points in a circle and a sphere, as well as the lemmas and theorem given in the paper, are of practical importance for solving the particular problems of forming a structure in group of UAVs. It can be assumed that quasi-uniformity in the general case also ensures the safety of the formation in the presence of wind loads. Thus, the proposed models of points' allocation can be applied for planning of UAV formations. The problem of assignment of UAVs to goal positions is formulated and considered.

This research was supported by the Russian Science Foundation (Project No. 17-71-10163).

\section{References}

1. D.Y. Ivanov, XII All-Russian conference on control problems, 1971-1978 (2014)

2. I.V. Vatamaniuk, G.Y. Panina, A.L. Ronzhin, Large Scale Syst. Control, 58, 285-305 (2015)

3. X. Yan, D. Sun, Handbook of Research on Design, Control, and Modeling of Swarm Robotics, 233-261 (IGI Global, 2016)

4. W. Ren, N. Sorensen. J. Rob, Autom. Syst., 4, 324333 (2008)

5. M.U. Khan, S. Li, Q. Wang, Z. Shao, ACM Trans. Auton. Adapt. Syst., 2, 1-22 (2016)

6. V.A. Erofeeva, O.N. Granichin, V.I. Kiyaev, Russ. SC Days, 285-305 (2016)

7. V.V. Atamanov, M.A. Kozachok, V.V. Trushkov, M.V. Khachumov, J. Neurocomp., 1, 73-76 (2009)

8. E.L. Altschuler, T.J. Williams, E.R. Ratner, R. Tipton, R. Stong, F. Dowla, F. Wooten, Phys. Rev. Lett., 78, (1997)

9. M.V. Khachumov, Sci. Tech. Inf. Process. 6, 310316 (2012)

10. N.J.A. Sloane, R.H. Hardin, T.S. Duff and J. H. Conway, Discrete Comput. Geom., 14, 237-259 (1995)

11. H. Kuhn, Nav. Res. Logist. 2, 83-97 (1955)

12. A.A. Dyachenko, Izvestiya SFedU. Engineering sciences, 3, 22-30 (2012)

13. I.A. Kalyaev, A.R. Gayduk, S.G. Kapustyan, Models and algorithms of collective control in groups of robots (Phismathlit, Moscow, 2009)

14. Y. Zhou, A. Raghavan, J.S. Baras, IEEE Conference on Decision and Control (2016)

15. N.S. Abramov, D.A. Makarov, M.V. Khachumov, Autom. Remote Control, 76, 1070-1080 (2015) 